\title{
Screening for mouth cancer: the pros and cons of a national programme
}

\author{
P. R. Brocklehurst ${ }^{* 1}$ and P. M. Speight ${ }^{2}$
}

\section{Key points}

Mouth cancer is an important condition with a poor survival rate that requires ongoing vigilance from all health professions, including those that might identify cases opportunistically.
Studies show that it is possible to detect relevant oral lesions with an acceptable degree of accuracy, but designing a trial to determine the effectiveness of mouth cancer screening is not feasible due to the low prevalence of the disease.
Current evidence only satisfies five of the 20 criteria required by the UK Screening Committee for a national programme, so more research is needed, particularly on developing tests and biomarkers, which will identify only those lesions that are most likely to be malignant or progress to malignancy.

The paper highlights the pros and cons of a national programme for screening for oral cancer. It provides an overview of screening methods and the metrics used to evaluate a screening programme. It then reviews the evidence for mouth cancer screening against the criteria of the United Kingdom National Screening Committee.

\section{Introduction}

The World Health Organisation has argued that the management of mouth cancer should be an integral part of national cancer control programmes. ${ }^{1}$ The prevention of mouth cancer can be classified into primary, secondary and tertiary programmes. While primary prevention describes a process that prevents a disease from developing, secondary prevention programmes generally seek to detect disease while it is localised or 'early'. Tertiary prevention seeks to mitigate the morbidity from established disease and to improve quality of life.

Screening is one method of early disease detection and is defined as 'the application of a test to people who are free of the disease in question, in order to identify those who may have the disease and to distinguish them from those who may not.'2 A screening programme is an on-going process of examination and referral, applied to a defined population at set intervals, and it must be differentiated from case-finding or early detection, which

'Director of NWORTH Clinical Trials Unit and Honorary Consultant in Dental Public Health, Bangor University, The Normal Site, Holyhead Road, Gwynedd, LL57 2PZ2; ${ }^{2}$ Professor Emeritus, School of Clinical Dentistry, University of Sheffield

${ }^{*}$ Correspondence to: Paul Brocklehurst

Email: p.brocklehurst@bangor.ac.uk

Refereed Paper. Accepted 15 May 2018

DOI: 10.1038/sj.bdj.2018.918 is usually a one-off process of clinical examination aimed at diagnosing specific lesions. Screening is also distinct from epidemiological studies that 'screen' populations to determine the prevalence of a disease or lesions.

Many countries set strict criteria for new screening programmes to be initiated, for example, the UK National Screening Committee now assesses the evidence for a new programme against 20 criteria. $^{3}$ There are more than 20 screening programmes which have already been implemented in the UK, but only three are for cancer: these are cervical, breast and bowel cancer. As can be inferred from Table 1, the criteria demand a high level of evidence, and many of the implemented programmes may not now satisfy all of the 20 criteria. As indicated in the right column, many of the criteria have not been met or have never been evaluated or addressed for mouth cancer screening.

Population screening programmes are of three main types. Mass screening describes a process whereby the whole population are screened, but this type of programme is rarely used. Most programmes are selective and target a subset of the population who are felt to be at highest risk, for example the screening of particular age groups of women for cervical cancer, or the over-60s for bowel cancer. The third type is opportunistic screening, where individuals are examined when they attend a healthcare professional for some other, often unrelated, purpose. This may be used, for example, to check blood pressure when attending a general medical practitioner, or could involve a mucosal examination when attending the dentist for a routine check.

\section{Making a case for screening mouth cancer}

The management of established lesions of mouth cancer is complex and multidisciplinary, often involving surgery and radiotherapy, and is therefore expensive. By contrast, small, early-stage cancers can often be treated by simple surgery, with less cost, lower morbidity and a better chance of cure. For example, the five-year survival for stage I mouth cancer has been shown to be $96 \%$ compared with $57 \%$ for late disease at stage IV. ${ }^{4}$ In addition, many cases of mouth cancer are preceded by a potentially malignant disorder (often a white patch or leukoplakia) providing a further opportunity to prevent disease progression. The mouth is also easy to examine and mouth lesions are relatively easy to detect. This all suggests that mouth cancer screening should be easy to implement and be an effective public health measure to prevent mouth cancer. It seems perplexing therefore that no country in the world has implemented a national mouth cancer screening programme, and at our present state of knowledge it is unlikely to happen. ${ }^{5}$ The following sections will try to summarise why this is the case. 


\section{Table 1 Criteria for the implementation of a screening programme ${ }^{3}$}

\section{The condition:}

\begin{tabular}{|c|c|c|}
\hline 1 & $\begin{array}{l}\text { Must be an important health problem. The epidemiology and natural history must be } \\
\text { understood and there must be a detectable latent asymptomatic or early symptomatic } \\
\text { phase }\end{array}$ & $?$ \\
\hline 2 & $\begin{array}{l}\text { All cost-effective primary prevention interventions should have been implemented where } \\
\text { possible }\end{array}$ & $?$ \\
\hline 3 & $\begin{array}{l}\text { If the disease involves a mutation, the natural history of people with this status should } \\
\text { be understood, including the psychological implications }\end{array}$ & $\mathrm{n} / \mathrm{a}$ \\
\hline \multicolumn{3}{|c|}{ The test: } \\
\hline 4 & Should be simple, safe and validated & $\checkmark$ \\
\hline 5 & $\begin{array}{l}\text { The distribution of test values should be known (for example, sensitivity and specificity) } \\
\text { and the criteria for a positive test should be agreed }\end{array}$ & $?$ \\
\hline 6 & Should be acceptable to the target population & $\checkmark$ \\
\hline 7 & $\begin{array}{l}\text { There should be an agreed policy and process for the further referral and diagnostic } \\
\text { investigation of individuals who test positive }\end{array}$ & $x$ \\
\hline 8 & $\begin{array}{l}\text { If the test is for a mutation, the methods for selection and process of review should be } \\
\text { clearly set out }\end{array}$ & N/A \\
\hline
\end{tabular}

\section{The intervention:}

9 Should be an effective treatment or intervention for patients found to have disease, and evidence that this early treatment leads to better outcomes

10 Should be evidence-based policies covering which individuals should be offered treatment and the appropriate treatment to be offered

\section{The screening programme:}

\begin{tabular}{l|l|c}
\hline 11 & $\begin{array}{l}\text { There must be evidence from RCTs that the screening programme is effective in reducing } \\
\text { mortality or morbidity }\end{array}$ & $\mathbf{x}$ \\
\hline 12 & Should be clinically, socially \& ethically acceptable & $\checkmark$ \\
\hline 13 & Benefit should outweigh any physical or psychological harm & $\checkmark$ \\
\hline 14 & The programme should be cost effective & $?$ \\
\hline
\end{tabular}

\begin{tabular}{l|l|l}
\hline 15 & $\begin{array}{l}\text { Clinical management of the condition and patient outcomes should be optimised in all } \\
\text { healthcare providers }\end{array}$ & $?$ \\
\hline 16 & All other options for managing the condition should have been considered & $?$ \\
\hline 17 & $\begin{array}{l}\text { There must be a plan for managing the programme, and agreed quality assurance } \\
\text { standards }\end{array}$ & $?$ \\
\hline 18 & $\begin{array}{l}\text { There must be adequate staffing and facilities for the programme and for referrals, } \\
\text { diagnosis and treatment }\end{array}$ & $?$ \\
\hline 19 & $\begin{array}{l}\text { Evidence-based information explaining the purpose and the positive and negative } \\
\text { aspects of the programme must be available to participants }\end{array}$ & $?$ \\
\hline 20 & $\begin{array}{l}\text { Screening intervals, eligibility for screening and the testing process should be scientifi- } \\
\text { cally justifiable to the public }\end{array}$ & $\mathbf{x}$ \\
\hline
\end{tabular}

$\checkmark$-criteria met: $\boldsymbol{x}$-criteria not met or never evaluated: ? - Criteria partially met

\section{Methods for the evaluation of a screening programme}

Before a test can be used in clinical practice its efficacy must be determined in experimental programmes that enable a calculation of sensitivity and specificity. A screening examination or test is not intended to be diagnostic, so when an individual is screened there are four potential outcomes (Table 2). A perfect screening test would correctly classify everyone in the population who has the disease as positive (TP) and all people without the disease as negative (TN). However, this never occurs, because the tests can never be absolutely accurate and so some individuals are falsely classified as positive (FP) or negative (FN). There is therefore a balance between the sensitivity and specificity of the screening test. The sensitivity is calculated as TP/TP+FN and is the proportion of the population who are correctly classified as having the disease.
The specificity is calculated as $\mathrm{TN} / \mathrm{TN}+\mathrm{FP}$ and shows the proportion of the population correctly classified as disease-free. TP $+\mathrm{FN}$ is the number of people in the population who have the disease and TN + FP is the number in the population who do not have the disease. There is no ideal value for sensitivity and specificity, while it is desirable to have a low number of false positives, to avoid unnecessary investigations and anxiety, it is also important not to miss disease, so false negatives must also be as low as possible. In general, tests aim to have a sensitivity and specificity in the order of $80 \%$ or more.

Once the sensitivity and specificity have been determined they can be used to form a summary receiver operating characteristic (ROC) curve, which graphically illustrates the ability of the test to distinguish positives from negatives (Fig. 1) The graph plots the true positive rate (sensitivity) against the false positive rate ( 1 - specificity). The efficacy of the test can be represented as the area under the curve (AUC). The dotted line represents chance that is, the test is no better than a toss of a coin at detecting true positives and true negatives (AUC of 0.5). The higher curve gives an AUC with a higher value. Values of 0.8 or greater are considered to be the minimum for an acceptable test.

\section{Screening for mouth cancer}

At the present time no test has been shown to be effective for the accurate detection of oral lesions in the context of a screening programme. ${ }^{6}$ The only properly tested health technology used in experimental and observational studies for the detection of oral potentially malignant lesions or mouth cancer is a conventional oral examination (COE). The results of ten studies are summarised in Table 3. ${ }^{7-16}$ In a Cochrane diagnostic test accuracy review, Walsh et al. ${ }^{17}$ examined the potential of $\mathrm{COE}$, vital rinsing, light-based detection, biomarkers and mouth self-examination (MSE) as methods for the detection of potentially malignant lesions and early mouth cancer in apparently healthy adults. The review only included studies that had calculated sensitivity and specificity, and excluded studies that did not follow-up on subjects assigned as negative by the screening test (making the calculation of false negatives impossible). The review found that the sensitivity of COE was variable, but was always greater than 0.70 , and that specificity was consistently greater than 0.90 . None of 
the other technologies tested were sufficiently well evaluated to determine their test accuracy. Overall, Walsh et al. concluded that COE had a satisfactory test performance with sensitivities and specificities similar to those reported for breast and cervical cancer screening programmes. They also highlighted that a number of studies utilised non-medical or dental, healthcare workers as screeners, without any significant change to the test performance results. ${ }^{12-14}$ This shows that trained healthcare workers or dental care professionals are equally able to examine the mouth and detect relevant lesions, and could fully participate in programmes for screening or early detection. This has been confirmed by a number of more recent studies that have evaluated lesion identification by trained health workers. ${ }^{18,19}$

These studies have shown that it is possible to detect relevant oral lesions with an acceptable degree of accuracy, and suggest that $\mathrm{COE}$ may be a valuable screening test. However, a more important consideration is to determine whether a mouth cancer screening programme using COE, actually works. The primary outcome measure for the clinical efficacy of a screening programme is whether or not it reduces mortality within the population - that is, the programme must be shown to save lives. A Cochrane systematic review of screening programmes found that there has only ever been one properly conducted study that has evaluated the clinical- or cost-efficacy of mouth cancer screening. ${ }^{20}$ This was a large clusterrandomised controlled trial carried out over a period of 15 years involving four rounds of screening, completed in 1998, 2002, 2004 and 2009. ${ }^{21-24}$ The screening programme was carried out in 13 municipalities in Kerala, South India, divided into seven test (screened) clusters ( $\mathrm{n}=96,517)$ and six control (not screened) clusters ( $\mathrm{n}=95,356)$. Non-medical, but trained, university graduates performed a COE to identify potentially malignant lesions (including leukoplakia, oral submucous fibrosis and lichen planus), and ulcers suspicious of malignancy. The gold standard outcome measure for the study was to determine mortality from mouth cancer in the test and control cohorts.

The Kerala study did not show a significant difference in mouth cancer mortality for the screened group (15.4/100,000 person-years) compared to the control group $(17.1 / 100,000$ person-years), with a RR of 0.88 (95\% CI 0.69-1.12). However, at the end of the 15-year period, they were able to demonstrate a statistically significant $24 \%$ reduction in mortality

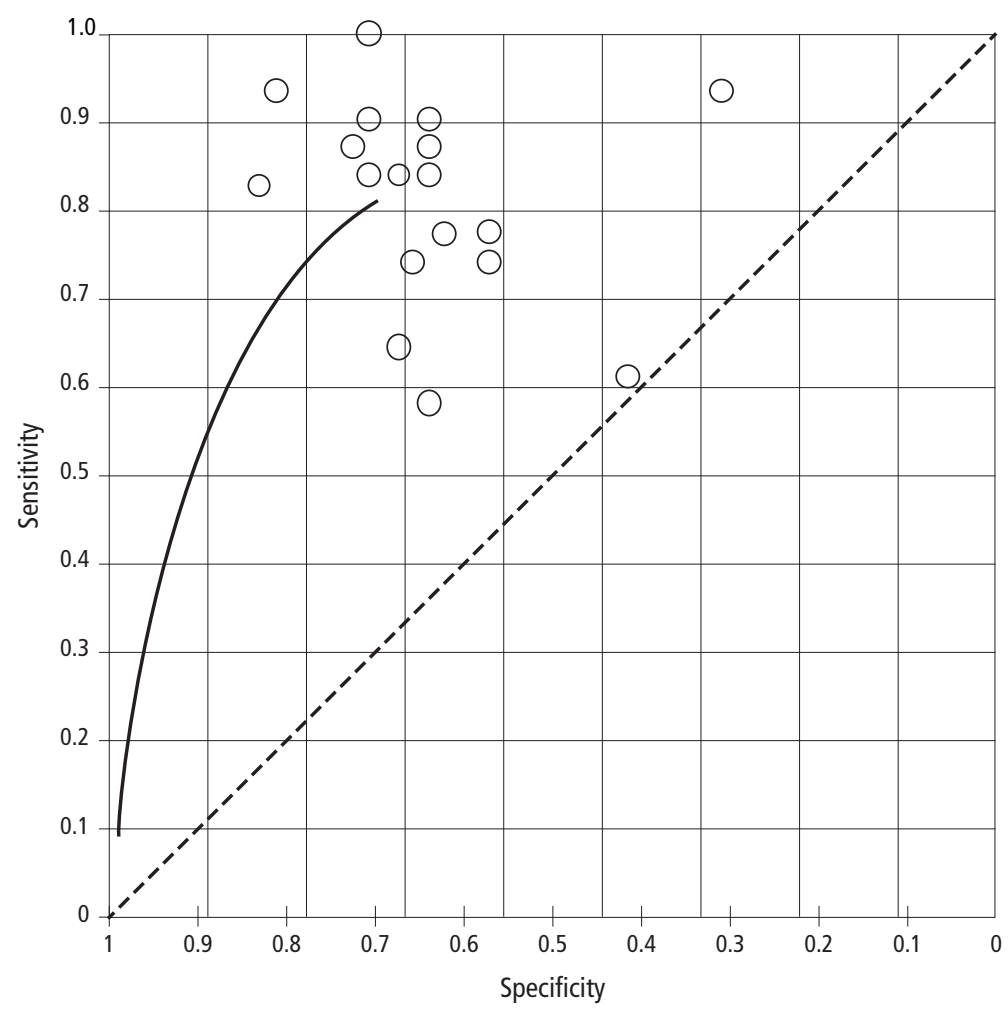

Fig. 1 Summary receiver operating characteristic curve

Table 2 Metrics for a screening programme

\begin{tabular}{l|l} 
Metric & Detail \\
\hline True positive (TP) & $\begin{array}{l}\text { Describes the outcome where someone with the disease in question is } \\
\text { correctly classified as being positive by the screening test }\end{array}$ \\
\hline False positive (FP) & When an individual is misclassified as positive, when in fact they are healthy \\
\hline True negative (TN) & $\begin{array}{l}\text { When the screening test correctly classifies a healthy person as not having } \\
\text { the disease in question }\end{array}$ \\
\hline False negative (FN) & $\begin{array}{l}\text { When an individual with the disease in question is classified by the screening } \\
\text { test as negative, in which case the lesions has been missed }\end{array}$ \\
\hline
\end{tabular}

for high-risk individuals who used tobacco and/or alcohol between the screened group (30/100,000 person-years) and the control group (39.0/100,000). ${ }^{25}$ Equally, it is important to note that only 19,288 persons completed four rounds of screening $-20 \%$ of the eligible population.

Further analysis of the Kerala programme ${ }^{26}$ found that the overall benefit of the screening programme was 269 life years saved per 100,000 for all individuals and 1,438 lives saved among the high-risk groups. The cost per screening examination was only $\$ 6$ per person and the incremental cost per life-year saved was US\$835 for all individuals and US $\$ 156$ for those at high-risk. In their systematic review, Brocklehurst et al. ${ }^{20}$ acknowledged the significant findings of the Kerala study, but also concluded that the trial had a high risk of bias, and concluded that further experimental evidence was needed to recommend population screening programmes. Similar conclusions were reached by Government bodies across the world and none have found the evidence sufficiently robust to recommend screening as a population health approach. ${ }^{5}$

\section{Should we screen for mouth cancer?}

The cost of screening demonstrated by the Kerala studies was within the target set by the WHO Commission on Macroeconomics and Health, who define an intervention as being cost effective when the cost-efficacy ratio is less than a country's gross domestic product per 
Table 3 Reports of evaluations of conventional oral examinations, in which sensitivity and specificity of the test have been calculated

\begin{tabular}{|c|c|c|c|c|c|c|}
\hline Study & $\mathrm{n}$ & $\%$ positive & Sensitivity & Specificity & PPV & NPV \\
\hline Chang et al. ${ }^{7}$ & 13,606 & 2.1 & 0.99 & 0.99 & - & - \\
\hline Downer et al. ${ }^{8}$ & 309 & 5.5 & 0.71 & 0.99 & 0.86 & 0.98 \\
\hline Ikeda et al. ${ }^{9}$ & 154 & 9.7 & 0.6 & 0.94 & 0.67 & 0.96 \\
\hline Jullien et al..$^{10}$ & 2027 & 2.7 & 0.74 & 0.99 & 0.67 & 0.99 \\
\hline Mathew et al."11 & 2069 & 10.3 & 0.94 & 0.98 & 0.87 & 0.99 \\
\hline Mehta et al..$^{12}$ & 1921 & 1.4 & 0.59 & 0.98 & 0.31 & 0.99 \\
\hline Warnakulasuriya et al. ${ }^{13}$ & 1872 & 21.6 & 0.95 & 0.81 & 0.58 & 0.98 \\
\hline Monteiro et al..$^{14}$ & 727 & 3.4 & 0.96 & 0.98 & 0.96 & 0.98 \\
\hline Nagao et al..15 & 137 & $68^{*}$ & 0.92 & 0.64 & 0.78 & 0.86 \\
\hline Sweeny et al. ${ }^{16}$ & 88 & 4.5 & 0.5 & 0.98 & - & - \\
\hline
\end{tabular}

capita. ${ }^{25}$ However, these values may not apply in a Western setting and to date there have been no randomised controlled trials in any developed country. This is mainly because of the low prevalence of relevant lesions making it very difficult and prohibitively expensive to conduct definitive trials of large scale population screening programmes for mouth cancer in countries such as the UK or USA.

An alternative approach was undertaken by Speight et al. ${ }^{26}$ who used computer modelling to simulate the costs of mouth cancer screening in a number of healthcare environments. Data to inform the model were taken from published NHS costs and from studies of disease prevalence and test performance. They found that mouth cancer screening in the UK would only be cost-effective if it were targeted at high-risk individuals and carried out opportunistically by GMPs and/or GDPs. In this scenario, the cost per life year saved was $£ 22,850$, which is within the costs deemed acceptable by NICE. ${ }^{26,27}$

These studies have shown that screening programmes have not resulted in a demonstrable reduction in mortality, although there is some evidence that screening of high-risk groups may be effective and cost-effective. At present the evidence is insufficient to persuade a government to sanction a national programme. In addition, there are other valid criticisms of screening for mouth cancer. Most of the studies that have evaluated a COE have used a lesion consistent with an mouth potentially malignant disorder or early mouth cancer as the criteria for a positive test. Most often this has been a white patch (leukoplakia), red patch (erythroplakia) or persistent ulcer, suggestive of cancer. Leukoplakia is the most common potentially malignant lesion, and although they are quite common, with a prevalence of between 1.4 and $22 \%,{ }^{28,29}$ the clinical appearance does not correlate well to histology and only about $5 \%$ overall will ever progress to cancer. ${ }^{30,31}$ This means that about $95 \%$ of screen detected lesions may not progress and are therefore not relevant to a test designed to detect lesions with a high likelihood of progressing to mouth cancer. The 'holy grail' is to identify adjunctive clinical tests or molecular methods that may identify the relevant $5 \%$. However, at the present time, no molecular biomarkers or adjunctive tests have been shown to have utility in screening trials. ${ }^{32,33}$

The management of oral potentially malignant disorders also remains a controversial area, raising doubt about the correct management for screen-detected lesions. Holmstrup et al. showed that even after surgical removal, the risk of malignant change may remain since the visible lesion may only represent a small area in a field of damaged mucosa. $^{34,35}$ As a result, even though early detection and intervention may remove the detected lesion, a whole field of damaged mucosa remains, that could progress to malignancy. There is also a lack of understanding about the malignant transformation of potentially malignant lesions and its temporal relationship with mouth cancer. ${ }^{30}$ Some potentially malignant lesions may become increasingly dysplastic or progress to cancer, but others remain static or may even regress. This lack of understanding of the detailed natural history of potentially malignant lesions, and the absence of molecular markers means that finding a test that can identify which lesions will become malignant remains a real challenge.

Finally, if opportunistic screening were an option, there remain questions about whether it would be effective in a dental practice setting. Clearly, dental healthcare workers are already trained to properly examine the mouth, but it has been suggested that patients at the highest risk of mouth cancer are the least likely to be regular attenders at dental practices. ${ }^{36}$ Further research is needed to undertake a retrospective analysis of cases to determine exactly the patient pathway to secondary care and the frequency and nature of contacts with healthcare professionals. This would help identify the potential nature and timing of appropriate interventions. Screening by non-medical or dental healthcare workers has been shown to be effective and utilising this group in alternative settings may be one of the most effective methods of improving early detection.

\section{Summary and conclusions}

As indicated above and illustrated in Table 1, studies into mouth cancer screening have only provided evidence to satisfy five of the 20 criteria required by the UK Screening Committee. The majority of issues have not been evaluated or have been shown not to meet the criteria. In some cases the criteria are partially met, but more research is needed. For example, there is little doubt that mouth cancer is an important health problem, but to satisfy criteria 1 , there needs to be more research into the natural history of the disease. In particular, we need to fully understand the biology of potentially malignant lesions and we need biomarkers that can be used to develop a test which is more specific than COE and will identify only those lesions which are most likely to progress to cancer (Criteria 5). For these reasons, mouth cancer screening has not been implemented. In the meantime, oral healthcare workers should remain vigilant when examining the mouth and be alert to the presence of mucosal changes which may be associated with potentially malignant disorders or early mouth cancer. In addition, patients should be instructed about high-risk habits including alcohol and tobacco use.

1. Petersen PE. Oral cancer prevention and control - the approach of the World Health Organization. Oral Oncol 2009; 45: 454-460.

2. Wilson J M, Jungner $Y$ G. Principles and practice of screening for disease. Public health paper number 34 WHO. Geneva, Switzerland, 1968.

3. UK National Screening Committee. Criteria for appraising the viability, effectiveness and appropriateness of 
a screening programme. 2015. Available at: https:// www.gov.uk/government/publications/evidencereview-criteria-national-screening-programmes/ criteria-for-appraising-the-viability-effectiveness-andappropriateness-of-a-screening-programme (accessed April 2018).

4. Rogers S N, Brown J S, Woolgar J A et al. Survival following primary surgery for oral cancer. Oral Oncol 2009; 45: 201-211.

5. Speight P M, Epstein J, Kujan 0 et al. Screening for oral cancer - A perspective from the Global Oral Cancer Forum. Oral Surg Oral Med Oral Pathol Oral Radiol 2016; 123: $680-687$

6. Lingen M W, Kalmar J R, Karrison T, Speight P M. Critical evaluation of diagnostic aids for the detection of oral cancer. Oral Oncol 2008; 44: 10-22.

7. Chang I H, Jiang R S, Wong Y K, Wu S H, Chen F J, Liu $S A$. Visual screening of oral cavity cancer in a male population: experience from a medical center. J Chin Med Assoc 2011; 74: 561-566.

8. Downer M C, Evans A W, Hughes Hallett C M, Jullien J A, Speight P M, Zakrzewska J M. Evaluation of screening for oral cancer and precancer in a company headquarters. Community Dent Oral Epidemiol 1995; 23: 84-88.

9. Ikeda N, Downer M C, Ishii T, Fukano H, Nagao T, Inoue K. Annual screening for oral cancer and precancer by invitation to 60 year-old residents of a city in Japan. Community Dent Health 1995; 12: 133-137.

10. Jullien J A, Downer M C, Zakrzewska J M, Speight P M Evaluation of a screening test for the early detection of oral cancer and precancer. Community Dent Health 1995; 12: 3-7.

11. Mathew B, Sankaranarayanan R, Sunilkumar K B, Kuruvila B, Pisani P, Nair M K. Reproducibility and validity of oral visual inspection by trained health workers in the detection of oral precancer and cancer. Br J Cancer 1997; 76: 390-394.

12. Mehta F S, Gupta P C, Bhonsle R B et al. Detection of oral cancer using basic health workers in an area of high oral cancer incidence in India. Cancer Detect Prev 1986; 9: 219-225.

13. Warnakulasuriya S, Pindborg J J. Reliability of oral precancer screening by primary health care workers in Sri Lanka. Community Dent Health 1990; 7: 73-79.
14. Monteiro L S, Salazar F, Pacheco J J, Martins M, Warnakulasuriya $\mathrm{S}$. Outcomes of invitational and opportunistic oral cancer screening initiatives in Oporto, Portugal. J Oral Pathol Med 2015; 44: 145-152.

15. Nagao $T$, Ikeda N, Fukano H, Miyazaki H, Yano M, Warnakulasuriya S. Outcome following a population screening programme for oral cancer and pre-cancer in Japan. Oral Oncol 2000; 36: 340-346.

16. Sweeny L, Dean N R, Magnuson J S, Carroll W R, Clemons L, Rosenthal E L. Assessment of tissue auto-fluores cence and reflectance for oral cavity cancer screening. Otolaryngol Head Neck Surg 2011; 145: 956-960.

17. Walsh T, Liu J L, Brocklehurst P et al. Clinical assessment to screen for the detection of oral cavity cancer and potentially malignant disorders in apparently healthy adults. Cochrane Database Syst Rev 2013; DOI: 10.1002/14651858.CD010173.pub2.

18. Brocklehurst P, Pemberton M N, Macey R, Cotton C, Wals $T$, Lewis M. Comparative accuracy of different members of the dental team in detecting malignant and non-malignant oral lesions. Br Dent J 2015; 218: 525-529.

19. Epstein J B, Gorsky M, Cabay R J, Day T, Gonsalves W. Screening for and diagnosis of oral premalignant lesions and oropharyngeal squamous cell carcinoma: role of primary care physicians. Can Fam Physician 2008; 54: 870-875.

20. Brocklehurst $P$, Kujan O, O'Malley L A, Ogden G, Shepherd S, Glenny A M. Screening programmes for the early detection and prevention of oral cancer. Cochrane Database Syst Rev 2013: CD004150.

21. Sankaranarayanan R, Mathew B, Jacob B J et al. Early findings from a community-based, cluster-randomized, controlled oral cancer screening trial in Kerala, India. The Trivandrum Oral Cancer Screening Study Group. Cancer 2000; 88: 664-673.

22. Ramadas K, Sankaranarayanan R, Jacob B J et al. Interim results from a cluster randomized controlled oral cancer screening trial in Kerala, India. Oral Oncol 2003; 39: 580-588.

23. Sankaranarayanan R, Ramadas $\mathrm{K}$, Thomas $\mathrm{G}$ et al. Trivandrum Oral Cancer Screening Study Group. Effect of screening on oral cancer mortality in Kerala, India: a cluster-randomised controlled trial. Lancet 2005; 365: 1927-1933.
24. Subramanian S, Sankaranarayanan R, Bapat B et al. Cost-effectiveness of oral cancer screening: results from a cluster randomized controlled trial in India. Bull World Health Organ 2009; 87: 200-206.

25. Commission on Macroeconomics and Health. Macroeconomics and health: investing in health for economic development. Geneva: World Health Organization, 2001

26. Speight P M, Palmer S, Moles D R et al. The cost-effectiveness of screening for oral cancer in primary care. Health Technol Assess 2006; 10: 1-144.

27. National Institute for Healthcare Excellence (NICE). Judging whether public health interventions offer value for money. 2013: https://www.nice.org.uk/advice/lgb10 (accessed October 2018).

28. Petti S. Pooled estimate of world leukoplakia prevalence: a systematic review. Oral Oncol 2003; 39: 770-780.

29. Lim K, Moles D R, Downer M C, Speight P M. Opportunistic screening for oral cancer and precancer in general dental practice: results of a demonstration study. $\mathrm{Br}$ Dent 2003: 194: 487-502.

30. Speight P M, Khurram S A, Kujan O. Oral potentially malignant disorders: risk of progression to malignancy. Oral Surg Oral Med Oral Pathol Oral Radiol 2017; 125: 612-627.

31. Napier S S, Speight P M. Natural history of potentially malignant oral lesions and conditions: an overview of the literature. J Oral Pathol Med 2008; 37: 1-10.

32. Warnakulasuriya S, Reibel J, Bouquot J, Dabelsteen E. Oral epithelial dysplasia classification systems: predictive value, utility, weaknesses and scope for improvement. J Oral Pathol Med 2008; 3: 127-133.

33. Macey R, Walsh T, Brocklehurst P et al. Diagnostic tests for oral cancer and potentially malignant disorders in patients presenting with clinically evident lesions. Cochrane Database Syst Rev 2015; DOI: 10.1002/14651858.CD010276.pub2

34. Holmstrup P. Can we prevent malignancy by treating premalignant lesions? Oral Oncol 2009; 45: 549-550.

35. Holmstrup P, Vedtofte P, Reibel J, Stoltze K: Oral premalignant lesions: is a biopsy reliable? J Oral Pathol Med 2007; 36: 262-266.

36. Netuveli G, Sheiham A, Watt R G. Does the 'inverse screening law' apply to oral cancer screening and regular dental check-ups? J Med Screen 2006; 13: 47-50. 